\title{
Fabrication and Characterization of Electrospun Wool Keratin/Poly(vinyl alcohol) Blend Nanofibers
}

\author{
Shuai Li and Xu-Hong Yang \\ National Engineering Laboratory for Modern Silk, College of Textile and Clothing Engineering, Soochow University, 199 Renai Road, \\ Suzhou 215123, China \\ Correspondence should be addressed to Xu-Hong Yang; yangxuhong@suda.edu.cn
}

Received 2 October 2013; Revised 4 December 2013; Accepted 17 December 2013; Published 9 January 2014

Academic Editor: Peter Chang

Copyright (C) 2014 S. Li and X.-H. Yang. This is an open access article distributed under the Creative Commons Attribution License, which permits unrestricted use, distribution, and reproduction in any medium, provided the original work is properly cited.

Wool keratin/poly(vinyl alcohol) (PVA) blend nanofibers were fabricated using the electrospinning method in formic acid solutions with different weight ratios of keratin to PVA. The resultant blend nanofibers were characterized by scanning electron microscopy (SEM), Fourier transform infrared (FTIR), X-ray diffraction (XRD), thermal gravimetric analysis (TGA), and tensile test. SEM images showed that the diameter of the blend nanofibers was affected by the content of keratin in blend solution. FTIR and XRD analyses data demonstrated that there were good interactions between keratin and PVA in the blended nanofibers caused by possibly hydrogen bonds. The TGA study revealed that the thermal stability of the blend nanofibers was between those of keratin and PVA. Tensile test indicated that the addition of PVA was able to improve the mechanical properties of the electrospun nanofibers.

\section{Introduction}

In recent years, electrospinning has drawn much attention because it is an easy, versatile, and low cost technique to fabricate nanofibers. In general, electrospinning apparatus is made up of a high voltage supply, a capillary tube with a pipette or needle of small diameter, and a metallic collector [1]. At present, a large number of organic and inorganic materials has been electrospun into nanofibers. As polymer nanofibers have high specific surface area and porosity, they are mainly used for filtration [2], environmental cleaning [3], tissue engineering [4], battery materials [5], and so forth. The study of electrospun biopolymers mainly focuses on silk fibroin [6], chitosan [7], and gelatin [8]. But keratin has received relatively poor attention.

Keratin is an abundant nonfood protein which is the major component of wool, hair, feathers, horns, and nails [9]. Keratin wastes, as poor quality raw nails from sheep breeding, by-products from the textile industry and horns, and nails and feathers from butchery [10], not only cause the waste of keratin resources but also pollute the environment. So, it is of great significance to study the recycling of keratin for effective utilization. Wool contains up to $95 \%$ by weight of pure keratin [11]. Compared with other proteins (e.g., fibroin and gelatin), wool keratin contains a large amount of cystine, which accounts for $7-20 \%$ of the total amino acids [12]. Wool keratin has a molecular weight ranging from 45 to $60 \mathrm{kDa}$ of the microfibrils from the cortical cells to $6-28 \mathrm{kDa}$ of the protein from the matrix [13]. At present, wool keratin has been prepared into films [14], sponge scaffolds [15], and nanofibers [16] and mainly used for biomedical application and adsorption. Wool keratin is difficultly electrospun into nanofibers because of its low molecular weight and brittle property. Some of the literature has reported the electrospinning of the blend of wool keratin with poly(ethylene oxide) [17], silk fibroin [18], polyamide 6 [19], and poly(lactic-co-glycolic acid) [20]. Recently, pure keratin has been electrospun without the addition of other polymers $[16,21,22]$. However, the average fiber diameter of the mats is larger than $200 \mathrm{~nm}$ and the breaking stress is not very high.

In this work, the blend of wool keratin and poly(vinyl alcohol) (PVA) was fabricated into nanofibers by electrospinning. Keratin/PVA nanofibers could be interesting candidates 
for preparing metal ion adsorbents because of the large number of functional group and high specific surface. The purpose of the use of PVA as an additive was to improve the processability of wool keratin and to enhance the mechanical properties of the blend nanofiber mats. Wool keratin and PVA with different weight proportions were mixed and dissolved in formic acid solution. The blend solutions were electrospun to produce nanofibers. The morphologies, structures, thermal stability, and mechanical properties of the blend nanofibers were investigated and correlated with the keratin/PVA blend ratios with the aim of highlighting the effect of the blend ratios on the structures and properties of nanofibers.

\section{Experimental}

2.1. Materials. The wool fibers were commercially obtained from wool textile factory. They were first cleaned by Soxhlet extraction with petroleum ether to remove fatty matter, then rinsed with distilled water and conditioned at $20^{\circ} \mathrm{C}$ and $65 \% \mathrm{RH}$ for $48 \mathrm{~h}$. Poly(vinyl alcohol) (PVA) with an average polymerization degree of 1700 and a saponification degree of 99\% was bought from Sinopharm Chemical Reagent Co., Ltd., China. All other chemicals used were of analytical reagent grade.

\subsection{Preparation of the Keratin/PVA Blend Solutions. Keratin} protein was extracted from wool by sulphitolysis with sodium metabisulphite according to the previous method [13]. Five grams of wool fibers were immersed in $100 \mathrm{~mL}$ of the urea $(8 \mathrm{M})$ and sodium metabisulphite $(0.5 \mathrm{M})$ mixture solution whose $\mathrm{pH}$ was adjusted to 6.5 using sodium hydroxide. The mixture was heated to $65^{\circ} \mathrm{C}$ and shaken for $2 \mathrm{~h}$ [23]. The resulting solution was filtered with a medium-speed qualitative filter paper. Afterwards, the filtrate was dialyzed against distilled water using a dialysis tube (molecular weight cutoff of 8,000-14,000 Da) for 3 days at room temperature. The solution obtained after dialysis was filtered with a medium-speed qualitative filter paper again. The filtrate was cast on a polyethylene plate at $50^{\circ} \mathrm{C}$ to obtain regenerated keratin.

The $12 \%$ wt solutions with the keratin/PVA weight ratios of 50/50,60/40, and 70/30 were prepared by dissolving wool keratin and PVA in formic acid ( $88 \% \mathrm{wt})$. The blend solutions were homogeneous without any precipitates and air bubbles.

2.3. Electrospinning of the Keratin/PVA Blend Nanofibers. The nanofibers were prepared by electrospinning in Nanofiber Electrospinning Unit (Kato Tech Co., Ltd., Japan). Electrospinning was performed at $20 \pm 3^{\circ} \mathrm{C}$ and $65 \pm 5 \% \mathrm{RH}$. The spinning parameters were as follows: voltage $20 \mathrm{kV}$, discharge speed $0.2 \mathrm{~mL} / \mathrm{h}$, and distance between syringe and collecting target $11 \mathrm{~cm}$.

2.4. Characterization. The rheological performance of the keratin/PVA solutions was measured by the AR2000 rheometer (TA Instruments, USA) with cone-plate geometry (40 mm diameter, $20^{\circ}$ angle); the shear rate was logarithmically increased from 0.1 to $1000 \mathrm{~s}^{-1}$ at $25^{\circ} \mathrm{C}$. The conductivity of each blend solution was measured by the DDS-11A electric conductivity meter (Shanghai INESA Scientific Instrument Co., Ltd., China).

The morphologies of the collected blend nanofibers were observed using the S-4800 scanning electron microscope (SEM) (Hitachi, Japan) after gold coating; the diameters of nanofibers were measured randomly by the image analysis software (Image-Pro Plus 5.0), and the average value was calculated from 100 measurements. The Fourier transform infrared spectra (FT-IR) of the nanofibers cut into powder were recorded with the Nicolet 5700 FT-IR spectrometer (Thermo Fisher Scientific Inc., USA) using potassium bromide pellets; all of the IR data were collected from 32 scans with a resolution of $4.0 \mathrm{~cm}^{-1}$. The crystal structures of the nanofibers were investigated by X-ray diffraction (XRD) using the $\mathrm{X}^{\prime}$ Pert PRO diffractometer (PANalytical B.V., The Netherlands) with a $\mathrm{CuK} \alpha$ radiation source operated at a tube voltage of $40 \mathrm{kV}$ and a tube current of $35 \mathrm{~mA}$; a diffraction range of $5^{\circ}-45^{\circ}(2 \theta)$ was selected and the scanning rate was $8^{\circ} / \mathrm{min}$. The thermal properties of the nanofibers were analyzed by the SDT Q600 thermogravimetric analyzer (TA Instruments, USA) under the conditions of a nitrogen flux of $100 \mathrm{~mL} / \mathrm{min}$, a heating rate of $10^{\circ} \mathrm{C} / \mathrm{min}$, a temperature range from 50 to $600^{\circ} \mathrm{C}$, and a sample weight of about $5 \mathrm{mg}$. The keratin/PVA nanofibers were cut into $40 \mathrm{~mm} \times 5 \mathrm{~mm}$. The thickness of these nanofiber mats was measured using a micrometer. Before using an automatic tensile tester (model 3365 electronic strength tester, Instron, Boston, USA) to characterize the mechanical properties of the mats, the samples were kept for $24 \mathrm{~h}$ at standard atmospheric conditions. During test process, distance between grips and test speeds were set to $20 \mathrm{~mm}$ and $10 \mathrm{~mm} \mathrm{~min}^{-1}$, respectively. An average of five measurements was reported for each sample.

\section{Results and Discussion}

3.1. Solution Characterization. Figure 1 shows the rheological behaviors of the keratin/PVA blend solutions with different keratin/PVA weight ratios, where the changes in viscosity as a function of shear rate are presented. The shape of the viscosity curves shows that with an increase in shear rate, all the solutions with the exception of keratin solution behaved as typical non-Newtonian, shear-thinning fluids. The viscosity curves of the keratin/PVA blend solutions were between those of pure keratin and PVA solutions in the entire range of measured shear rates. It is worth noting that the rheological behaviors were dependent on solution composition. Compared to the solutions rich in wool keratin, the solutions with a higher percentage of PVA showed a greater change in viscosity with increasing shear rate and had stronger non-Newtonian nature. In other words, the pseudoplasticity of the solutions increased with an increase in the proportion of PVA in the blends, which endues the keratin and PVA blend fluid with good flow behaviors.

The viscosity is an important factor for the electrospinning of keratin/PVA blend solution and fiber formation. As shown in Figure 2, the zero-shear viscosity estimated from 


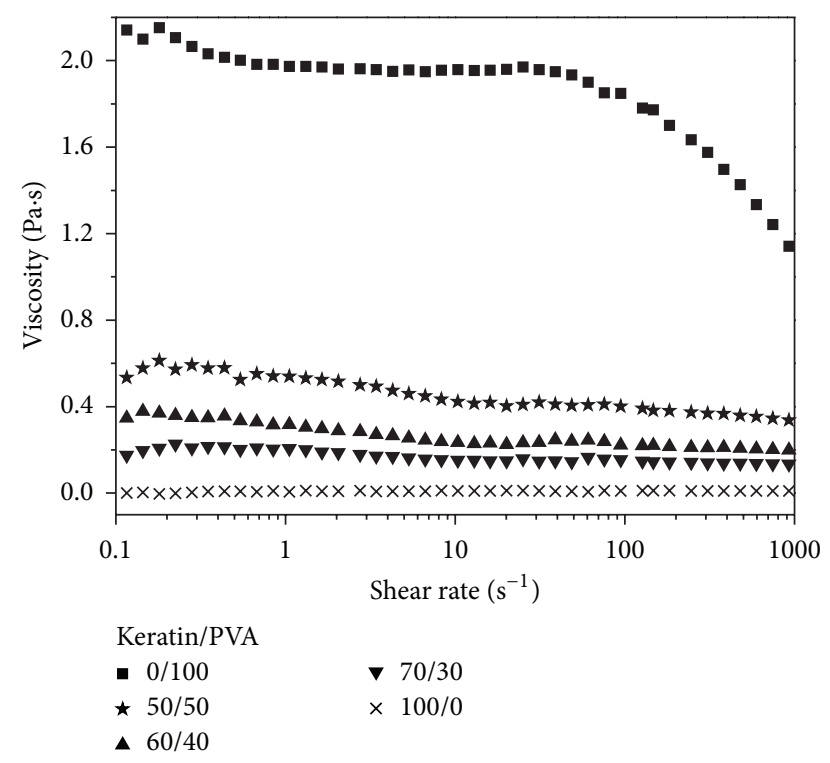

FIGURE 1: Rheological behaviors of the keratin/PVA blend solutions.

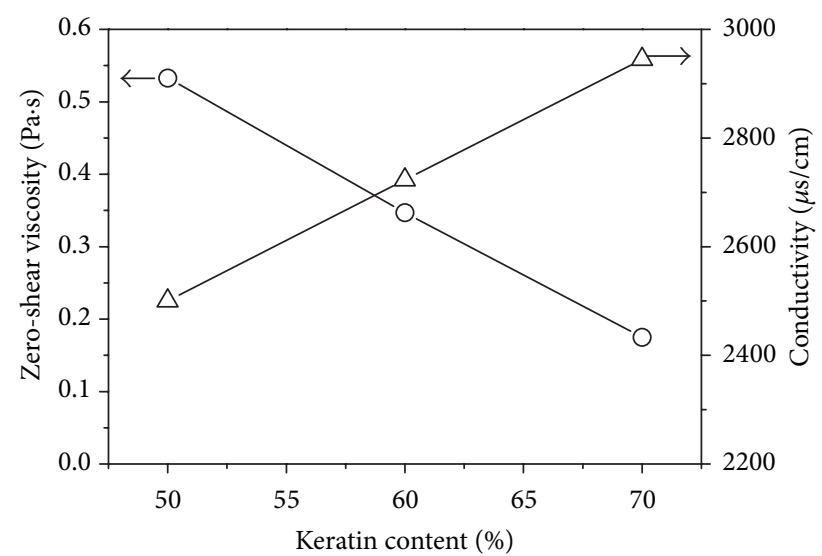

FIGURE 2: Zero-shear viscosity and conductivity of the keratin/PVA blend solutions as a function of keratin content.

the flow curve decreased with increasing keratin content, which is caused by the reduction in the concentration of entangled macromolecules in solution. Figure 2 also shows that the conductivity of blend solutions increased with increasing keratin content because a large number of amino acids in keratin was readily ionized in formic acid solution [24]. In a certain range of polymer concentrations, the increasing solution conductivity can promote the fiber formation [17]. That means that during the jet process, the higher the conductivity of the solution is, the larger the electrostatic spinning drafting force is; therefore the nanofiber is easy to form.

3.2. Morphologies. Figure 3 shows the SEM micrographs of the keratin/PVA blend nanofibers. From these micrographs, it is evident that the surface of the blend nanofibers was smooth and had no bead defect. Moreover, the phaseseparated morphology was not observed from the blend nanofibers. Figure 4 shows that the average diameters of the blend nanofibers decreased from $83 \mathrm{~nm}$ to $69 \mathrm{~nm}$ when the content of keratin in spinning solution increased from $50 \%$ to $70 \%$. The decrease in the average diameters could be explained by the reduction in the viscosity and the increase in the conductivity of spinning solution with increasing keratin content. Meanwhile, the diameter distribution became significantly narrower when the keratin content increased.

3.3. FT-IR Spectra. Figure 5 shows the FT-IR spectra of keratin powder, electrospun PVA, and keratin/PVA blend nanofibers. The spectrum of pure keratin which was not capable of being electrospun was measured using the film sample prepared by casting its formic acid solution at $50^{\circ} \mathrm{C}$. For the spectrum of keratin, the absorption bands at 1650 , 1539 , and $1232 \mathrm{~cm}^{-1}$ were the characteristic amide peaks of amide I, II, and III, respectively [25]; the strong broad peak at $3415 \mathrm{~cm}^{-1}$ was characteristic of the $\mathrm{N}-\mathrm{H}$ stretching vibration, and the intense peak at $1025 \mathrm{~cm}^{-1}$ indicated the S-O symmetric stretching vibration of cysteine-S-sulfonate residues $[20,23]$. The electrospun PVA fiber exhibited the peaks from the secondary hydroxyl group, including $\mathrm{O}-\mathrm{H}$ stretching vibration at $3523 \mathrm{~cm}^{-1}, \mathrm{O}-\mathrm{H}$ deformation vibration at $1441 \mathrm{~cm}^{-1}$, and $\mathrm{C}-\mathrm{O}$ stretching vibration at $1181 \mathrm{~cm}^{-1}$ [7]. The absorption peaks at $1717 \mathrm{~cm}^{-1}$ of the PVA and keratin blend nanofibers were attributed to the presence of the carbonyl group which belongs to residual formic acid. It was observed that with the increase of PVA content in the blends, the intensities of the absorption bands at 1650, 1539, and $1025 \mathrm{~cm}^{-1}$ relative to keratin content decreased as expected. It is worth mentioning that in the case of the addition of PVA, the absorption peak of keratin at about $3415 \mathrm{~cm}^{-1}$ shifted to a lower wave number and became much broader. This strongly suggested the formation of hydrogen bond between keratin and PVA molecules. Therefore the inclusion of PVA could moderate the interaction between keratin macromolecules and thus improve the electrospinnability of keratin with PVA.

3.4. XRD Analyses. Figure 6 presents the XRD patterns of keratin powder, electrospun PVA, and keratin/PVA blend nanofibers. The keratin powder showed a small peak at about $9.5^{\circ}(2 \theta)$ and a very broad peak at $20.5^{\circ}(2 \theta)$, corresponding to the $\beta$-sheet structure $[20,23]$. The electrospun PVA nanofibers showed a significant crystalline peak at about $23.5^{\circ}$ $(2 \theta)$, which is due to the occurrence of strong intermolecular and intramolecular hydrogen bonding. If there was no or weak interaction between keratin and PVA molecules in the blend fibers, each component would have its own crystal region in the blend fibers and the XRD patterns would be expressed as the simple mixture of keratin and PVA patterns. In fact, the peak of keratin at $9.5^{\circ}$ disappeared in the blend. The diffraction peak positions of the keratin/PVA blend fibers were located between those of keratin and PVA diffraction peaks. These evidences further suggested that strong interactions occurred between keratin and PVA molecules in the blend. In addition, the intensity of diffraction showed that the blend nanofibers exhibited the degree of crystallinity similar to that of the electrospun PVA fiber, implying the 


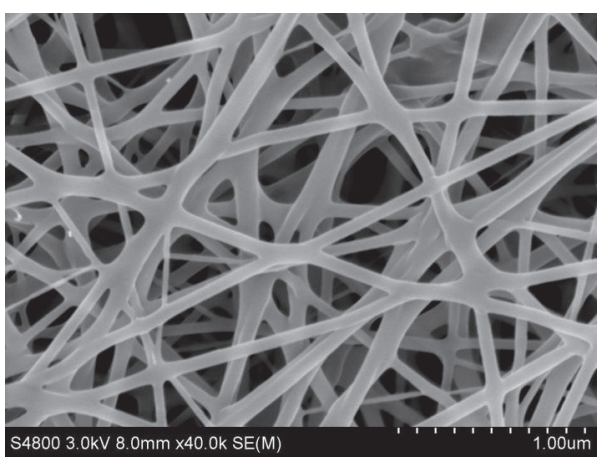

(a)

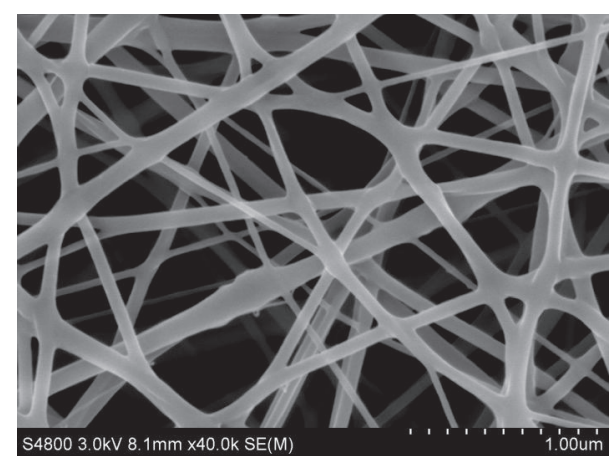

(b)

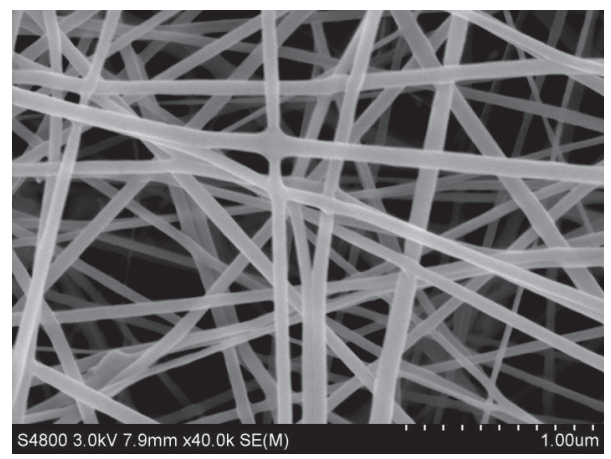

(c)

FIGURE 3: SEM micrographs of the blend nanofibers obtained using various weight ratios of keratin to PVA: (a) 50/50, (b) 60/40, and (c) $70 / 30$.

main contribution of PVA to the crystallinity of the blend nanofibers. According to the above discussions, it can be concluded that the addition of PVA enables the improvement of the electrospinnability and fiber formation ability of the keratin/PVA blend.

3.5. Thermal Behaviors. Figure 7 shows the thermal properties of keratin powder, electrospun PVA, and keratin/PVA blend nanofibers. In the case of keratin, the initial weight loss corresponding to moisture vaporization occurred below $180^{\circ} \mathrm{C}$, and the second weight loss caused by the decomposition of keratin molecules took place in the temperature range from 200 to $400^{\circ} \mathrm{C}$ [23]. In the TGA curves of PVA, three weight loss peaks were observed. The first weight loss took place below $190^{\circ} \mathrm{C}$ due to moisture loss, the second and major weight loss at $200-300^{\circ} \mathrm{C}$ due to the thermal degradation of PVA, and the third weight loss at $400-450^{\circ} \mathrm{C}$ due to the by-product formation of PVA during thermal degradation $[26,27]$. Keratin exhibited higher onset temperature, slower speed, and higher weight residue of thermal degradation than PVA and therefore higher thermal stability. The TGA curves of the blend nanofibers were located between those of keratin and PVA and their shape approached to that of keratin. In the light of the onset temperature, speed and, weight residue of thermal degradation, the thermal stability of the blend nanofibers ranged from those of keratin and PVA and increased with increasing keratin content.
3.6. Mechanical Properties. Figure 8 shows the representative stress-strain curves of the electrospun nanofiber mats. The average breaking stress of keratin/PVA 50/50, 60/40, and $70 / 30$ was $9.22,6.02$, and $6.50 \mathrm{MPa}$, respectively. The average breaking strains was $48 \%, 28 \%$, and $30 \%$, respectively. The average Young modulus was $1.39,1.38$, and $1.18 \mathrm{MPa}$, respectively. The results exhibited that the breaking stress and break strain of the mats of keratin/PVA 50/50 were far higher than those of keratin/PVA 60/40 and 70/30. This can be explained that the higher PVA content in nanofiber mats enhanced the mechanical properties of the mats. The result of Young modulus also showed the trend that Young modulus increased with the increasing PVA content. The breaking stress and break strain of the mats of keratin/PVA 60/40 are slightly lower than those of keratin/PVA 70/30. This could be due to the unevenness mat structure of keratin/PVA 60/40.

\section{Conclusions}

In this study, the wool keratin/PVA blend nanofibers were successfully prepared by electrospinning. The addition of PVA was able to overcome the poor electrospinnability of keratin. SEM micrographs showed that the blend nanofibers had smaller and narrower diameter distributions with increasing keratin content, and their diameter was below $100 \mathrm{~nm}$. The results of FTIR and XRD indicated that there were strong interactions between keratin and PVA. The thermal stability 


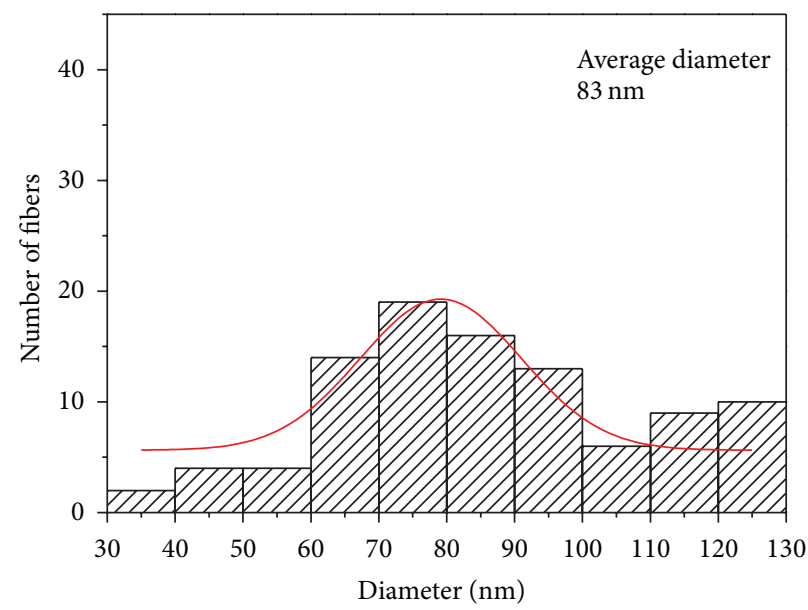

(a)

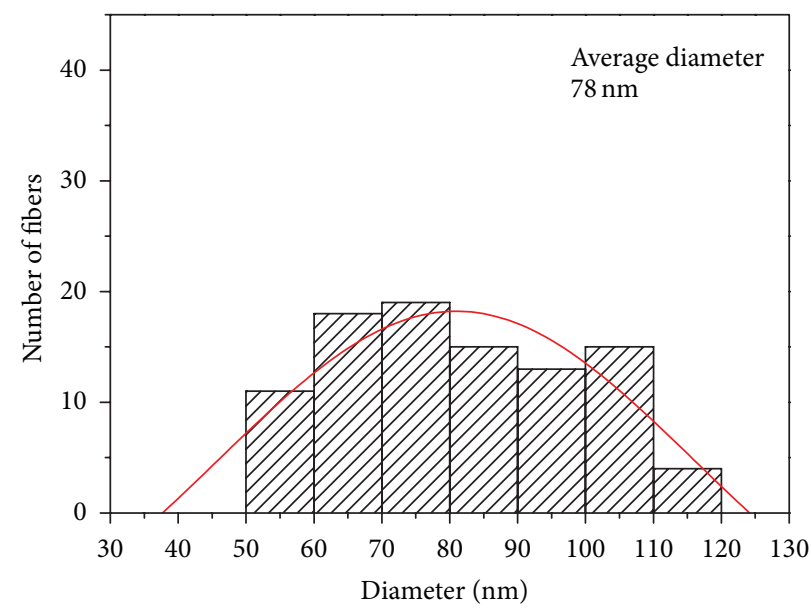

(b)

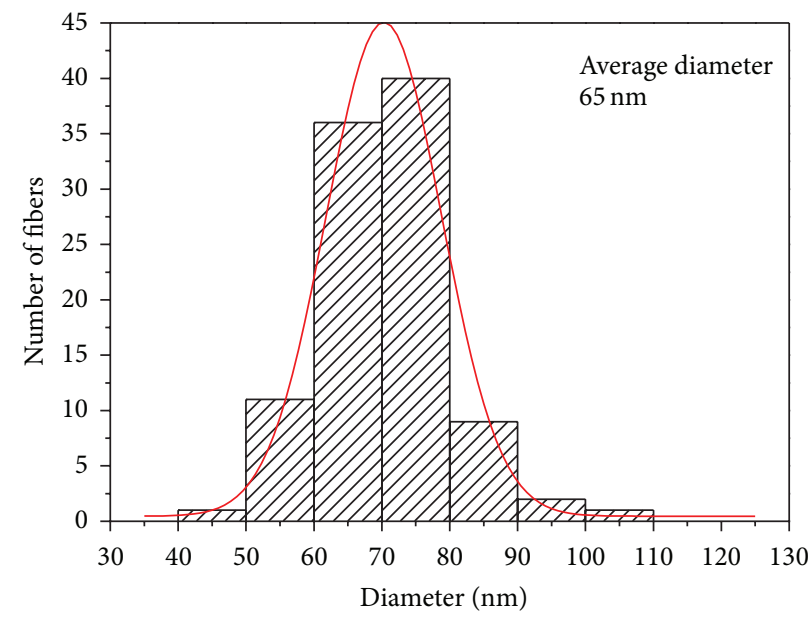

(c)

FIGURE 4: Diameter distribution of the blend nanofibers obtained using various weight ratios of keratin to PVA: (a) 50/50, (b) 60/40, and (c) $70 / 30$.

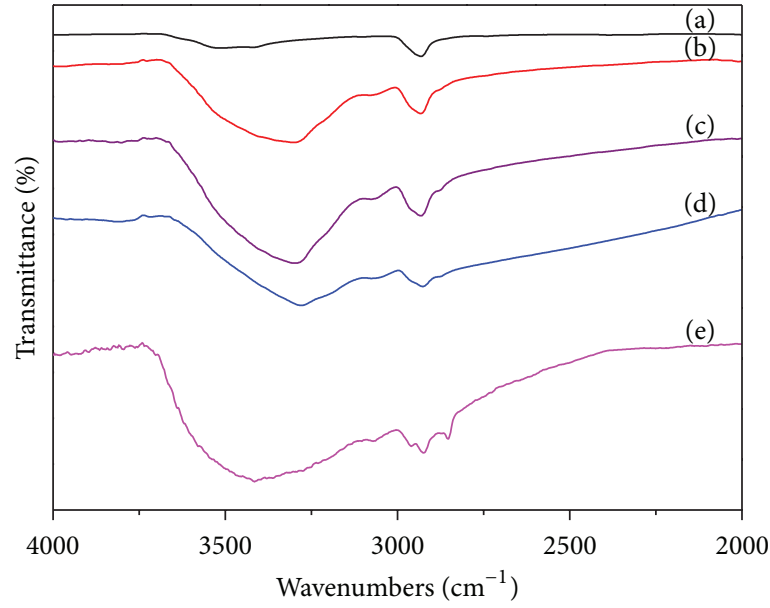

(a)

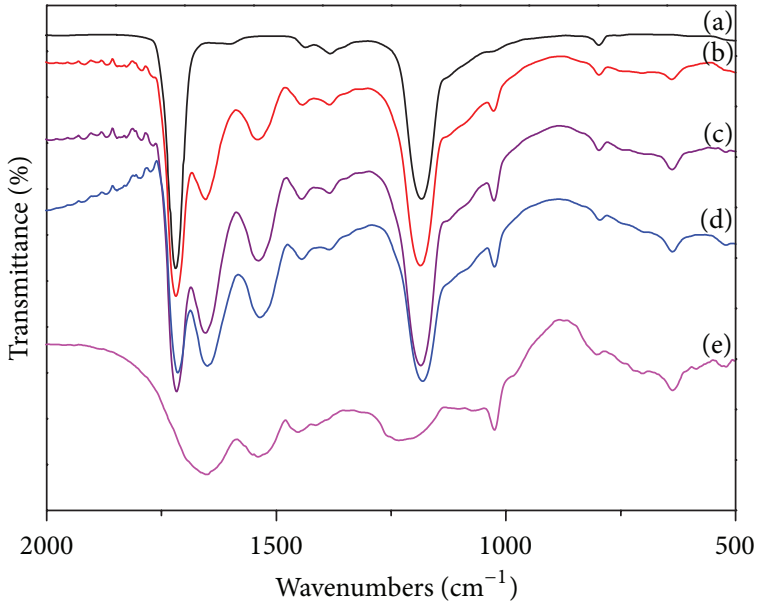

(b)

FIGURE 5: FTIR spectra of (a) electrospun PVA and (e) keratin powder as well as the blend nanofibers obtained using various weight ratios of keratin to PVA: (b) 50/50, (c) 60/40, and (d) 70/30. 


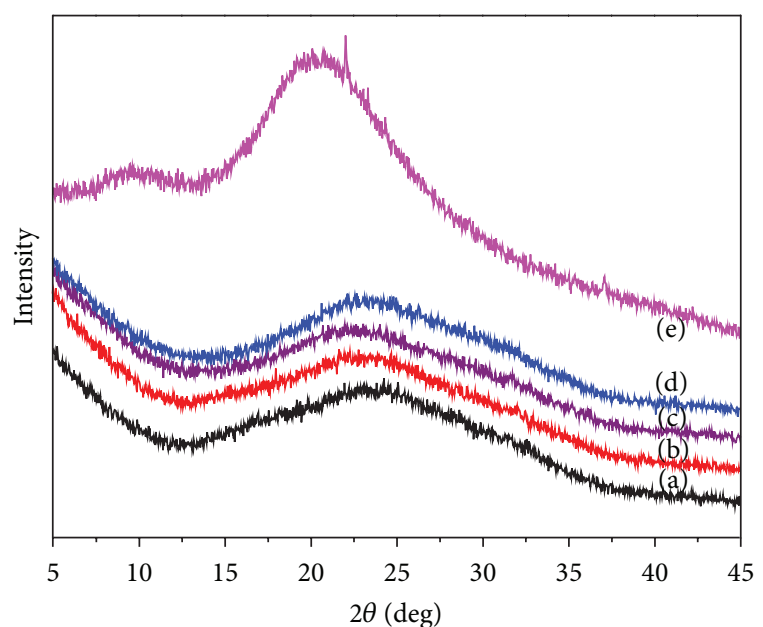

Electrospun fibers: keratin/PVA
(a) $0 / 100$
(d) $70 / 30$
(b) $50 / 50$
(c) $60 / 40$
(e) Keratin powder

FIGURE 6: XRD patterns of (a) electrospun PVA and (e) keratin powder as well as the blend nanofibers obtained using various weight ratios of keratin to PVA: (b) 50/50, (c) 60/40, and (d) 70/30.

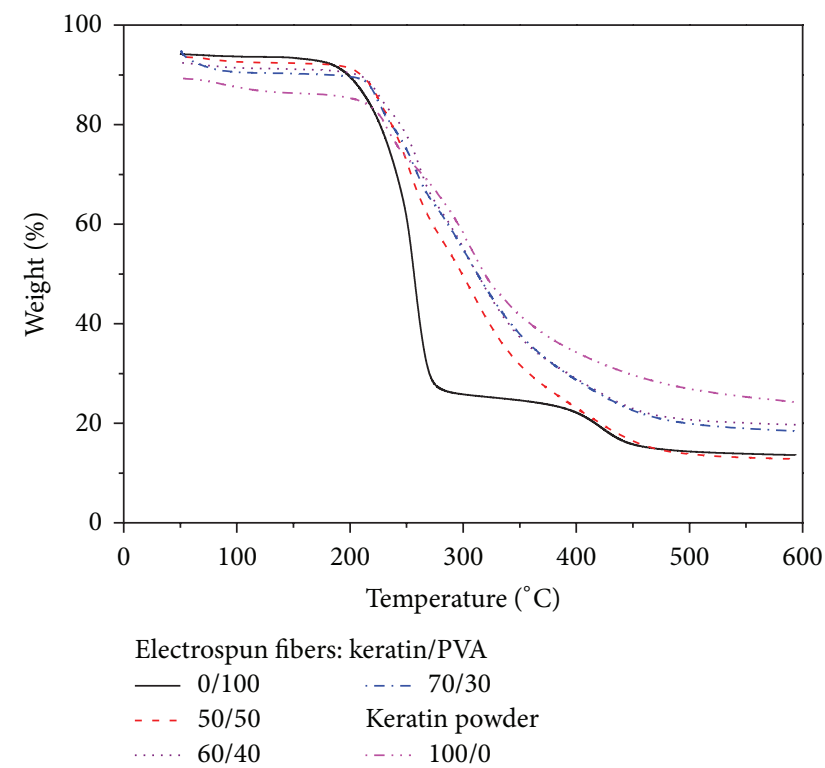

FIGURE 7: TGA data of electrospun PVA, keratin powder, and the blend nanofibers obtained using various weight ratios of keratin to PVA.

of the blend nanofibers ranged from those of keratin and PVA and increased with increasing keratin content. The results of tensile test showed that the addition of PVA was able to improve the mechanical properties of the electrospun nanofiber mats.

\section{Conflict of Interests}

The authors declare no conflict of interests.

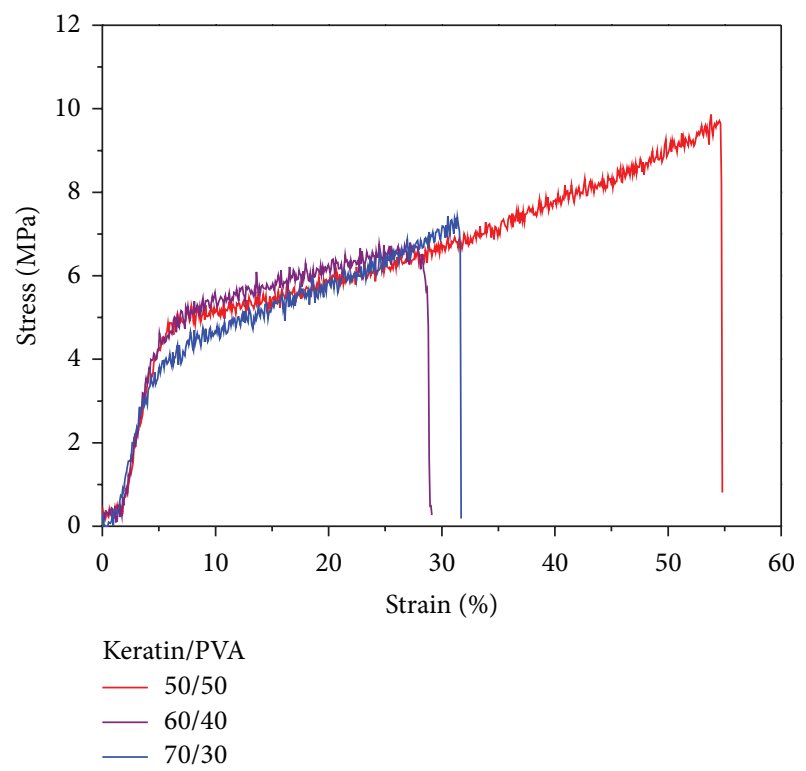

FIGURE 8: Stress-strain curves of the blend nanofibers obtained using various weight ratios of keratin to PVA.

\section{Acknowledgments}

This study was funded by the Priority Academic Program Development (PAPD) of Jiangsu Higher Education Institutions and the Technology Innovation Foundation of National Engineering Laboratory for Modern Silk.

\section{References}

[1] T. Inami, Y. Tanimoto, M. Ueda, Y. Shibata, and S. Hirayama, "Morphology and in vitro behavior of electrospun fibrous poly(D,L-lactic acid) for biomedical applications," Advances in Materials Science and Engineering, vol. 2013, Article ID 140643, 6 pages, 2013.

[2] R. Gopal, S. Kaur, Z. Ma, C. Chan, S. Ramakrishna, and T. Matsuura, "Electrospun nanofibrous filtration membrane," Journal of Membrane Science, vol. 281, no. 1-2, pp. 581-586, 2006.

[3] K. Saeed, S. Haider, T.-J. Oh, and S.-Y. Park, "Preparation of amidoxime-modified polyacrylonitrile (PAN-oxime) nanofibers and their applications to metal ions adsorption," Journal of Membrane Science, vol. 322, no. 2, pp. 400-405, 2008.

[4] C. Huang, R. Chen, Q. Ke, Y. Morsi, K. Zhang, and X. Mo, "Electrospun collagen-chitosan-TPU nanofibrous scaffolds for tissue engineered tubular grafts," Colloids and Surfaces B, vol. 82, no. 2, pp. 307-315, 2011.

[5] A. R. S. Priya, A. Subramania, Y.-S. Jung, and K.-J. Kim, "High-performance Quasi-solid-state dye-sensitized solar cell based on an electrospun PVdF-HFP membrane electrolyte," Langmuir, vol. 24, no. 17, pp. 9816-9819, 2008.

[6] F. Zhang, B. Q. Zuo, H. X. Zhang, and L. Bai, "Studies of electrospun regenerated SF/TSF nanofibers," Polymer, vol. 50, no. 1, pp. 279-285, 2009.

[7] Y.-T. Jia, J. Gong, X.-H. Gu, H.-Y. Kim, J. Dong, and X.-Y. Shen, "Fabrication and characterization of poly (vinyl alcohol)/chitosan blend nanofibers produced by electrospinning method," Carbohydrate Polymers, vol. 67, no. 3, pp. 403-409, 2007. 
[8] D. Yang, Y. Li, and J. Nie, "Preparation of gelatin/PVA nanofibers and their potential application in controlled release of drugs," Carbohydrate Polymers, vol. 69, no. 3, pp. 538-543, 2007.

[9] R. D. B. Fraser, T. P. MacRae, and G. E. Rogers, Keratin: Their Composition, Structure and Biosynthesis, Charles C. Thomas, Springfield, Ill, USA, 1972.

[10] E. Salminen and J. Rintala, "Anaerobic digestion of organic solid poultry slaughterhouse waste-a review," Bioresource Technology, vol. 83, no. 1, pp. 13-26, 2002.

[11] P. V. Peplow and A. D. Rodick-lanzilotta, "Orthopaedic materials derived from keratin," United States Patent 2005/0232963 A1, 2005.

[12] L. M. Dowling, W. G. Crewther, and A. S. Inglis, "The primary structure of component $8 \mathrm{c}-1$, a subunit protein of intermediate filaments in wool keratin. Relationships with proteins from other intermediate filaments," Biochemical Journal, vol. 236, no. 3, pp. 695-703, 1986.

[13] J. A. Maclaren and B. Milligan, Wool Science: The Chemical Reactivity of the Wool Fiber, Science Press, Marrickville, Australia, 1981.

[14] K. Katoh, M. Shibayama, T. Tanabe, and K. Yamauchi, "Preparation and physicochemical properties of compression-molded keratin films," Biomaterials, vol. 25, no. 12, pp. 2265-2272, 2004.

[15] K. Katoh, T. Tanabe, and K. Yamauchi, "Novel approach to fabricate keratin sponge scaffolds with controlled pore size and porosity," Biomaterials, vol. 25, no. 18, pp. 4255-4262, 2004.

[16] A. Aluigi, C. Vineis, C. Tonin, C. Tonetti, A. Varesano, and G. Mazzuchetti, "Wool keratin-based nanofibres for active filtration of air and water," Journal of Biobased Materials and Bioenergy, vol. 3, no. 3, pp. 311-319, 2009.

[17] A. Aluigi, A. Varesano, A. Montarsolo et al., "Electrospinning of keratin/poly(ethylene oxide) blend nanofibers," Journal of Applied Polymer Science, vol. 104, no. 2, pp. 863-870, 2007.

[18] M. Zoccola, A. Aluigi, C. Vineis, C. Tonin, F. Ferrero, and M. G. Piacentino, "Study on cast membranes and electrospun nanofibers made from keratin/fibroin blends," Biomacromolecules, vol. 9, no. 10, pp. 2819-2825, 2008.

[19] A. Aluigi, C. Tonetti, C. Vineis, C. Tonin, and G. Mazzuchetti, "Adsorption of copper(II) ions by keratin/PA6 blend nanofibres," European Polymer Journal, vol. 47, no. 9, pp. 1756-1764, 2011.

[20] H. Zhang, "Electrospun poly (lactic-co-glycolic acid)/multiwalled carbon nanotubes composite scaffolds for guided bone tissue regeneration," Journal of Bioactive and Compatible Polymers, vol. 26, no. 4, pp. 347-362, 2011.

[21] A. Aluigi, A. Corbellini, F. Rombaldoni, and G. Mazzuchetti, "Wool-derived keratin nanofiber membranes for dynamic adsorption of heavy-metal ions from aqueous solutions," Textile Research Journal, vol. 83, no. 15, pp. 1574-1586, 2013.

[22] A. Aluigi, A. Corbellini, F. Rombaldoni, M. Zoccola, and M. Canetti, "Morphological and structural investigation of woolderived keratin nanofibres crosslinked by thermal treatment," International Journal of Biological Macromolecules, vol. 57, pp. 30-37, 2013.

[23] A. Aluigi, M. Zoccola, C. Vineis, C. Tonin, F. Ferrero, and M. Canetti, "Study on the structure and properties of wool keratin regenerated from formic acid," International Journal of Biological Macromolecules, vol. 41, no. 3, pp. 266-273, 2007.

[24] A. Aluigi, C. Vineis, A. Varesano, G. Mazzuchetti, F. Ferrero, and C. Tonin, "Structure and properties of keratin/PEO blend nanofibres," European Polymer Journal, vol. 44, no. 8, pp. 24652475, 2008.
[25] J.-S. Li, Y. Li, X. Liu, J. Zhang, and Y. Zhang, "Strategy to introduce an hydroxyapatite-keratin nanocomposite into a fibrous membrane for bone tissue engineering," Journal of Materials Chemistry B, vol. 1, no. 4, pp. 432-437, 2013.

[26] M. S. Islam and M. R. Karim, "Fabrication and characterization of poly(vinyl alcohol)/alginate blend nanofibers by electrospinning method," Colloids and Surfaces A, vol. 366, no. 1-3, pp. 135140,2010

[27] B. J. Holland and J. N. Hay, "The thermal degradation of poly(vinyl alcohol)," Polymer, vol. 42, no. 16, pp. 6775-6783, 2001. 

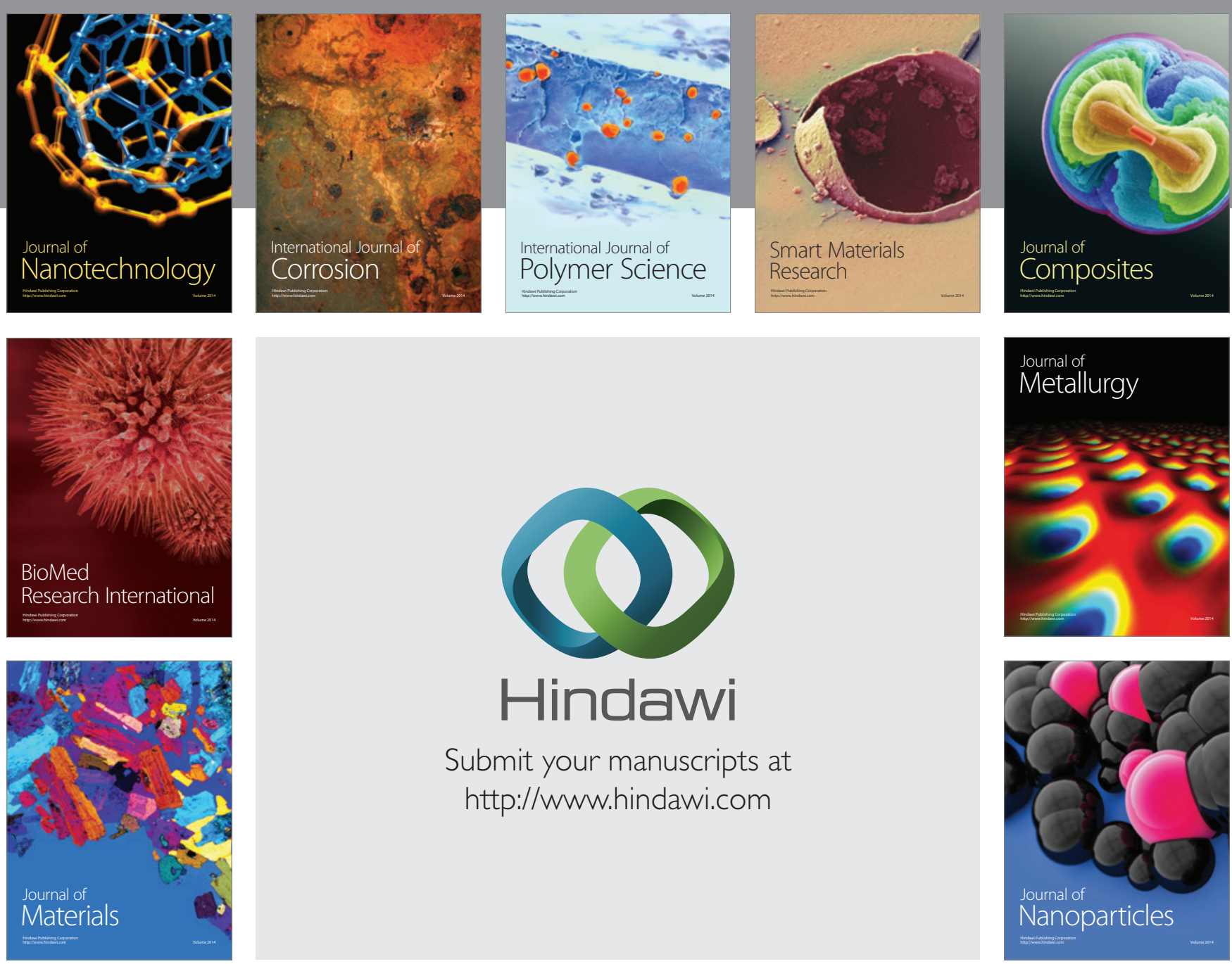

Submit your manuscripts at http://www.hindawi.com
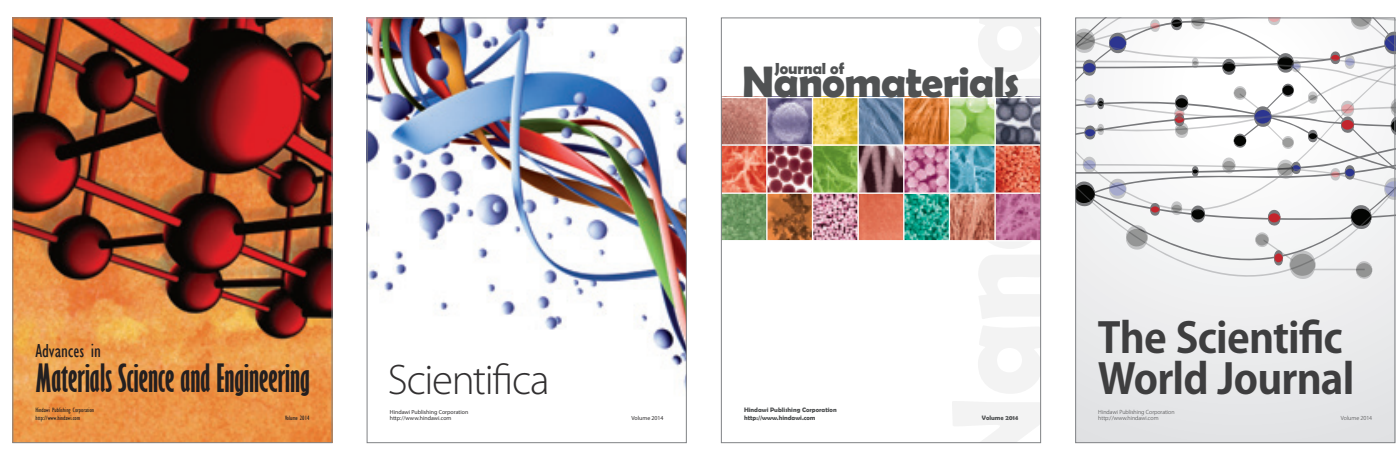

\section{The Scientific World Journal}
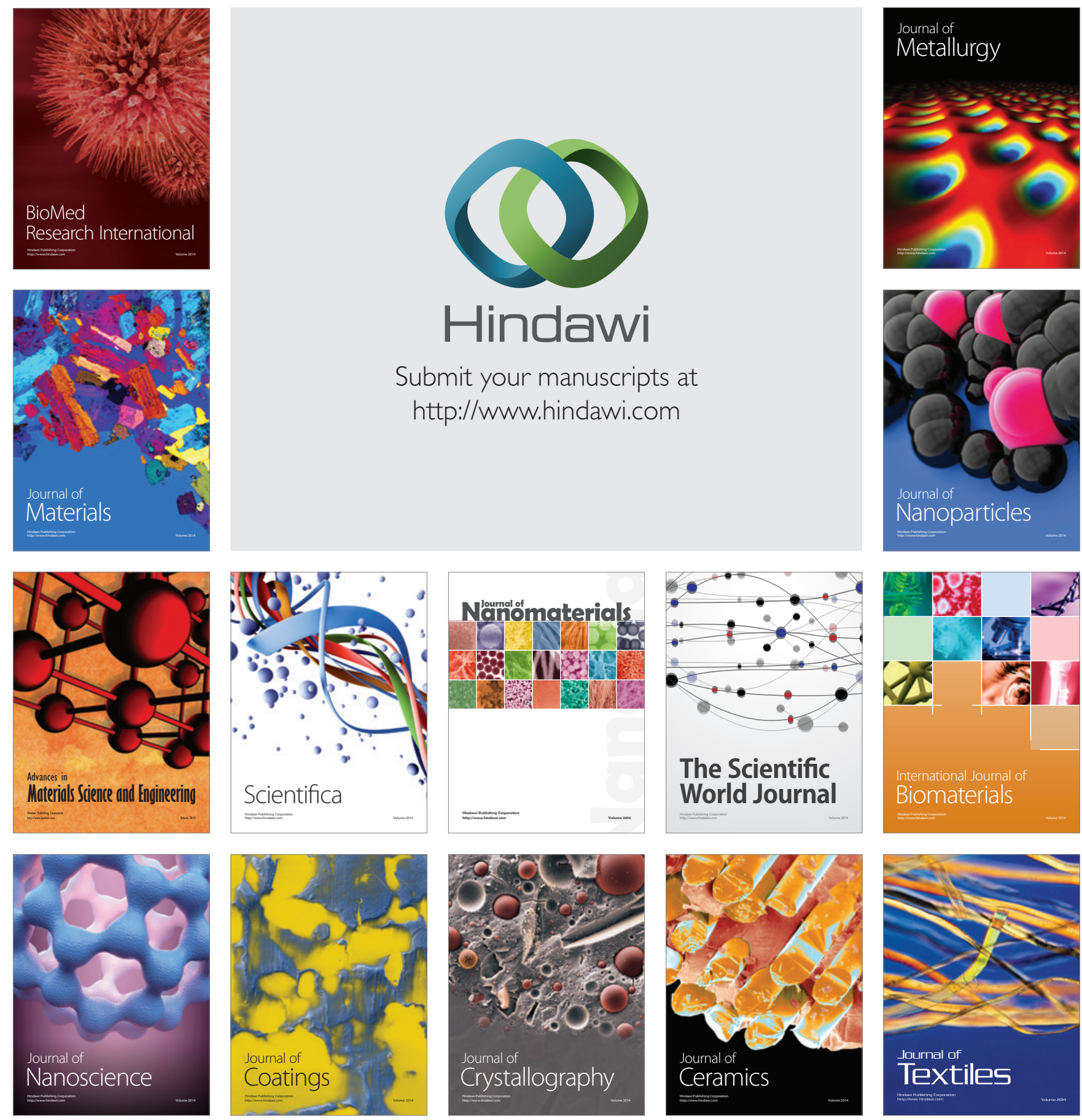